\title{
BRIGHT FEATURES IN THE INTERGRANULAR REGION
}

\author{
Z. SUEMOTO and E. HIEI \\ National Astronomical Observatory \\ Mitaka, Tokyo 181 \\ Japan
}

\begin{abstract}
There are two kinds of bright threads in spectrograms of the $\mathrm{Ca} \mathrm{II} \mathrm{H}$ and $\mathrm{K}$ lines: the ones in the outer line wing are due to enhancements of the continuum inside granules, while the others in the inner wing are due to bright features in the intergranular regions.
\end{abstract}

\section{Introduction}

It has generally been believed that granulation is the only inhomogeneity of the normal photosphere inside the supergranulation cells. In a paper by Suemoto et al. (1987) we have suggested the possible existence of another inhomogeneity (bright features) in the upper photosphere, which shows up as a bright continuum (provisionally called $\mathrm{K}_{0}$-continuum) in the inner wing ( $3 \AA>\Delta \lambda>0.5 \AA$ ) of the $\mathrm{Ca}$ II $\mathrm{K}$ line, and appears in the intergranular lanes. The granular continuum and the $\mathrm{K}_{0}$-continuum are confirmed to be located more or less complementarily. Further the absorption lines are found to be weakened at the location of the $\mathrm{K}_{0}$-continuum.

\section{Material and Reduction}

Spectrograms with a wavelength range from $3885 \AA$ to $4015 \AA$ were taken in one exposure in quiet regions at disk center with a linear dispersion of $0.56 \AA \mathrm{mm}^{-1}$. Two spectrograms were selected to be raster scanned with an aperture of $50 \mu \mathrm{m} \times 50 \mu \mathrm{m}(0.32 \operatorname{arcsec} \times 28 \mathrm{~m} \AA)$ and a step size of $20 \mu \mathrm{m} \times 20 \mu \mathrm{m}(0.13 \operatorname{arcsec} \times 11 \mathrm{~m} \AA)$ from $3918 \AA$ to $3985 \AA$. The total area of $24 \mathrm{~mm} \times 120 \mathrm{~mm}$ of the spectrogram gives $1200 \times 6000$ data points.

The contrast $C\left(\lambda_{j}, k\right)$ at a particular position $k$ along the slit at wavelength $\lambda_{j}$ is defined as the ratio between the intensity at that position and the mean intensity averaged over all positions along the slit at that wavelength. We selected 13 'windows' in the wings of the $\mathrm{Ca}$ II $\mathrm{H}$ and $\mathrm{K}$ lines, and derived 13 contrast curves along the slit, one for each window. 


\section{Results}

\subsection{SIZE AND DISTRIBUTION}

The widths of the granular continuum and the $\mathrm{K}_{0}$-continuum are inferred from their autocorrelation curves. The full width at half maximum of the granular continuum was estimated from the first minimum, and is about $1000 \mathrm{~km}$. It is to be noted here that the size of the $\mathrm{K}_{0}$-continuum is also about the same as that of granules.

Correlation coefficients of all the combinations of the 13 windows were calculated and are shown in Figure 1. The ordinates and the abscissae are the correlation coefficients from -1.1 to 1.0 , and the effective wavelength distance from the Ca II K line center, $\Delta \lambda_{k}^{*}$, respectively. The anti-correlation between the granular continuum and the $\mathrm{K}_{0}$-continuum is clearly seen.

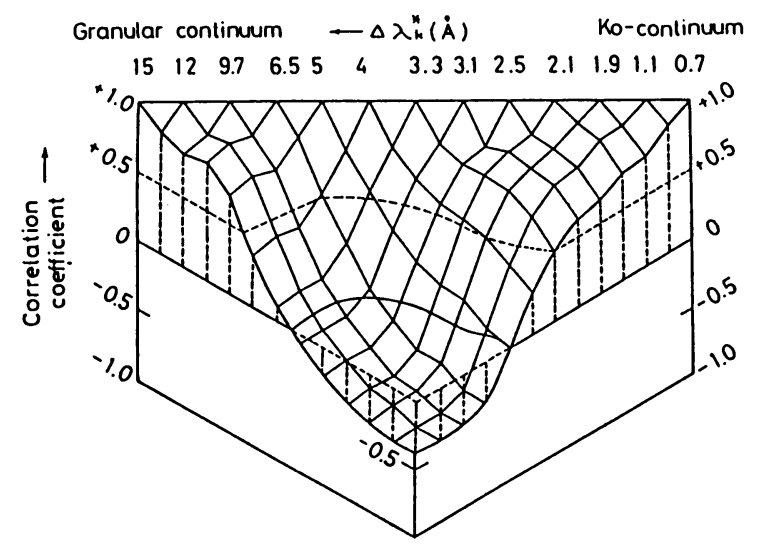

Figure 1. Correlation coefficient diagram for the brightness fluctuations. The correlation coefficients of all the combinations of $\Delta \lambda_{k}^{*}$ 's are plotted, where $\Delta \lambda_{k}^{*}$ is the effective wavelength distance from the $\mathrm{Ca}$ II $\mathrm{K}$ line center.

It has long been believed that the visibility of the granulation decreases towards the limb but persists far beyond $\cos \theta=0.3$ until the pattern finally becomes invisible. Edmonds (1962) measured the relative RMS of the brightness fluctuation of the granulation across the solar disk, which is characterized by a slow increase from disk center $\left(\theta=0^{\circ}\right)$ to $\theta \approx 50^{\circ}$, and a sharp decrease to $\theta \approx 70^{\circ}$, and then an increase again to $\theta \approx 74^{\circ}(\theta$ is the heliocentric angle). Keil (1977) also measured the center-to-limb variation of the RMS granular contrast which decreases monotonically between $\theta=0^{\circ}$ and $\approx 55^{\circ}$ and then increases slightly at $\theta \approx 70^{\circ}$. Beyond $\theta \approx 70^{\circ}$ there are various estimates of the distance from the limb at which the granulation disappears: $15-10$ arcsec (Edmonds, 1962), $10-4 \operatorname{arcsec}$ (Rösch, 1957; Loughead and Bray, 1960), and less than 2 arcsec (Pravdjuk et al., 1974; Muller, 1977). Although the distance of the disappearance is different among the authors, all the photographs show the persistence of the so-called 'granular pattern' towards the extreme limb.

Since the granular continuum does not extend much beyond the 'transition wavelength' towards the $\mathrm{K}$ line center, many granular regions could not extend much higher than $\tau \approx 0.3$. 
On the other hand, by similar reasoning, intergranular hot features would be located much higher than $\tau \approx 0.1$ in the photosphere. It is very likely, then, that what appears to be the granular pattern seen near the limb, say, around a heliocentric angle of $\theta \approx 70^{\circ}$, may be a mixture of normal granules and the bright features in the intergranular regions. The intensity at $\cos \theta \approx 0.2$ is about 0.3 times that at the disk center, which is equal to the residual intensity at the 'transition wavelength' mentioned above. Beyond $\theta \approx 70^{\circ}$ the bright features in the intergranular regions may become more and more important. Thus a higher value of the relative RMS of the brightness fluctuations beyond $\theta \approx 74^{\circ}$ derived by Edmonds (1962) might be due to the increasing predominance of the $\mathrm{K}_{0}$-continuum.

\subsection{CENTRAL INTENSITIES OF ABSORPTION LINES}

Since the $\mathrm{K}_{0}$-continuum is located much higher than, say, $\tau \approx 0.1$ in the photosphere, the central intensities of strong absorption lines may also be affected by the higher temperature responsible for the bright features of the $\mathrm{K}_{0}$-continuum.

The central intensities of strong lines clearly have a positive correlation with the $\mathrm{K}_{0}$ continuum, while the correlation is only slightly positive for intermediately strong lines. This is in good agreement with the suggestion mentioned above: central intensities of strong absorption lines, which are formed in the layer of the upper photosphere where the $\mathrm{K}_{0^{-}}$ continuum also originates, would become bright, but medium strong lines are formed at a somewhat lower height than that of the $\mathrm{K}_{0}$-continuum and the correlation becomes slightly worse. These correlations suggest that the source function of both the lines and the $\mathrm{K}_{0^{-}}$ continuum should have a hump in the upper layer of the photosphere in intergranular regions.

The correlations with the granular continuum are random for strong lines, while being positive for intermediately strong lines. This indicates that the strong lines have almost no relation with granules, while the central intensities of the intermediately strong lines change with the continuous background. This suggests that the source function of the lines and the continuum above granules should decrease monotonically with height, and that the high temperature zone may not extend to the upper layer of the photosphere.

Keil and Canfield (1978) studied the correlation between the central intensities of absorption lines and the granular continuum: the correlation is good for weaker lines, but becomes worse and even negative for the strongest lines, in good agreement with our results.

\subsection{RADIAL VELOCITY}

The line shift is defined as the central wavelength of the half-level chords of the absorption lines. The radial velocity is derived from the difference between the line shift at each position along the slit and the value averaged over the slit length.

There are good correlations between the line shifts and both continua, which means that upward velocities are predominant above the granules and downward velocities below the layer where the $\mathrm{K}_{0}$ bright features exist. This result is consistent with previous investigations (Krat and Shpitalnaya, 1974; Bray et al., 1984; Muller, 1985). 


\section{References}

Bray, R.J., Loughead, R.E., and Durrant, C.J. (1984), The Solar Granulation, 2nd edition, Cambridge Univ. Press.

Edmonds, F.N. Jr. (1962) Astrophys. J. Suppl. 6, 357.

Keil, S.L. (1977) Solar Phys. 53, 359.

Keil, S.L. and Canfield, R.C. (1978) Astron. Astrophys. 70, 169.

Krat, V.A. and Shpitalnaya, A.A. (1974) Solnechnye Dannye No.2, 63.

Muller, R. (1977) Solar Phys. 52, 249.

Muller, R. (1985) Solar Phys. 100, 237.

Pravdjuk, L.M., Karpinsky, V.N., and Andreiko, A.V. (1974) Solnechnye Dannye No.2, 70. Rösch, J. (1957) l'Astronomie 71, 129.

Suemoto, Z., Hiei, E., and Nakagomi, Y. (1987) Solar Phys. 112, 59. 